\title{
Endovascular treatment in patients with carotid artery dissection and intracranial occlusion: a systematic review
}

\author{
Jan W. Hoving ${ }^{1}$ - Henk A. Marquering ${ }^{1,2}$ - Charles B. L. M. Majoie ${ }^{1}$
}

Received: 16 January 2017 / Accepted: 15 May 2017 / Published online: 3 June 2017

(C) The Author(s) 2017. This article is an open access publication

\begin{abstract}
Purpose Recently, multiple randomised controlled trials showed efficacy of endovascular treatment over traditional care in patients with acute ischemic stroke due to an intracranial anterior circulation occlusion. Internal carotid artery (ICA) dissection with a concomitant intracranial occlusion is a rare but important cause of severe acute ischemic stroke. Although this subtype of acute ischemic stroke is mostly treated with endovascular treatment, treatment outcomes are still sparsely studied. This study assesses the clinical outcome and reperfusion rates by means of a systematic review.

Methods Electronic databases of PubMed, EMBASE and Web of Science were searched up to October 1, 2016 for articles describing endovascular treatment in patients with intracranial artery occlusion and ICA dissection.

Results Sixteen studies were included in the analysis. Most studies showed favourable outcome and successful reperfusion. However, most included studies had a high risk of bias. Conclusion In the reviewed studies, endovascular treatment in patients with ICA dissection and concomitant proximal intracranial occlusion was associated with favourable outcome. This could point in the direction of endovascular
\end{abstract}

Electronic supplementary material The online version of this article (doi:10.1007/s00234-017-1850-y) contains supplementary material, which is available to authorized users.

Jan W. Hoving

j.w.hoving@amc.uva.nl

1 Department of Radiology, Academic Medical Center, University of Amsterdam, Amsterdam, The Netherlands

2 Department of Biomedical Engineering and Physics, Academic Medical Center, University of Amsterdam,

Amsterdam, The Netherlands treatment being a beneficial treatment method for these patients. However, this review has only taken data of a limited group of patients into account. A pooled analysis of patients from recently published endovascular treatment trials and running registries is therefore recommended.

Keywords Stroke $\cdot$ Internal carotid artery $\cdot$ Dissection . Thrombectomy $\cdot$ Stent

\section{Introduction}

Internal carotid artery (ICA) dissection is a major cause of acute ischemic stroke (AIS) in the young, responsible for $10-25 \%$ of all strokes occurring in this population [1], whereas this severe cause of AIS only account for up to $2 \%$ in the total stroke population [2]. Before the introduction of intraarterial therapy, only few patients with extracranial ICA dissection together with an intracranial vessel occlusion were reported to fully recover after intravenous administration of rtPA [3]. Over the past years, multiple studies on alternative ways of treatment such as intravenous fibrinolysis combined with endovascular treatment have been performed $[4,5]$. As a result of the publication of multiple randomised controlled trials (MR CLEAN, ESCAPE, REVASCAT, SWIFT PRIME, EXTEND-IA, THRACE and PISTE) [6-12], intraarterial treatment has been proven effective over traditional treatment and has become the new standard for exclusive proximal intracranial occlusions. However, although patients with ICA dissection in combination with concomitant intracranial vessel occlusion are mostly treated with endovascular treatment, the clinical outcome and reperfusion rates are still sparsely studied, and therefore, this type of treatment is still unproven to be beneficial. 
In this review, we assessed outcome for patients with ICA dissection in combination with intracranial vessel occlusion after use of endovascular treatment. We searched the available literature for cases describing patients with ICA dissection in combination with intracranial occlusion who received endovascular treatment and analysed clinical outcome and reperfusion rates.

\section{Methods}

\section{Literature search}

The online databases of PubMed, EMBASE and Web of Science were searched for data up to October 1, 2016. We used the following key words and MeSH terms: 'stroke', 'thrombectomy', 'carotid dissection', 'intraarterial', 'endovascular treatment' and 'endovascular therapy'. After completion of the search, the filter 'Humans' was used for all of the articles found in the three databases. Articles not written in English were excluded from the search.

\section{Study selection}

To meet the inclusion criteria for this systematic review, studies should have described patients with both ICA dissection and proximal intracranial occlusion, either as total sample of patients or as a subgroup, were included.

Subsequently, full-text screening of the articles was performed. Studies that only described patients with only internal carotid artery occlusion, exclusive ICA dissection, vertebrobasilar occlusion or dissection and patients who had exclusively received intravenous thrombolysis were excluded. Studies without specific dissection data, useful outcome parameters and description of the performed endovascular procedure were also not included in this review. Finally, conference abstracts were excluded.

\section{Data extraction}

Data on study design, year of publication, country of origin, number of included patients and type of procedure were extracted. Patient characteristics such as gender, age and locations of both extracranial dissection together with the intracranial occlusion were recorded. We also extracted the following data: time to treatment (TTT), outcome rates graded as 90-day mRS, outcome rates graded as 90-day NIHSS and reperfusion and graded with the TICI score [13]. Reperfusion data was divided into the following subgroups: TICI $0-1$, TICI $2 \mathrm{a}$ and TICI $2 b-3$.

To clearly denote the procedures for ICA dissection and intracranial occlusion that have been performed, the treatment options are represented in several subclasses. Procedures of
ICA dissection treatment were divided into two subclasses: (1) permanent stent placement in ICA dissection and (2) no stenting of the ICA dissection. Procedures of intracranial occlusion treatment were divided into four subclasses: (1) use of intraarterial (IA) thrombolytics, (2) use of a Merci retriever, (3) use of a Penumbra device and (4) use of a Solitaire stent retriever. Finally, any complications or unexpected additional interventions during treatment were noted.

\section{Assessment of quality}

The risk of bias was determined using an adapted version of the Cochrane Collaboration's Risk of Bias Tool [14]. This tool represents a domain-based evaluation, which critically assesses various domains. These domains are sequence generation, blinding of participants and personnel, blinding of outcome assessment, incomplete outcome data, selective outcome reporting and 'other issues'. The risk of bias for each domain was classified as 'high', 'low' or 'unclear'.

\section{Study outcomes}

In the studies included in this review, reperfusion was graded with the TICI score [13]. Accordingly, we defined TICI $2 b-3$ as successful reperfusion. In cases where reperfusion was described as favourable, we defined this as TICI $2 b$ to prevent overestimation of reperfusion rates. Functional outcome was graded with 90-day mRS and 90-day NIHSS. When studies described both parameters, we chose to present both 90-day NIHSS and 90-day mRS to preserve all found outcome data. Favourable outcome was defined as a mRS score $\leq 2$ and a NIHSS score $\leq 1$ or improvement by at least 11 points from baseline [15].

\section{Results}

\section{Search results}

The initial search resulted in 351 studies. After removing 29 duplicates and 32 articles that were not accessible online, 290 articles remained. After screening on title and abstract, 254 articles were excluded based on relevancy, leaving 36 articles for full-text screening. Finally, 16 articles were included in this literature review. Most frequent reasons for exclusion were no clear description of the use of endovascular treatment and absence of clear outcome data.

\section{Study characteristics}

Results of the risk of bias analysis are presented online as a supplementary table and as supplementary figures (see Online Resources 1, 2 and 3). All included studies were retrospective 
case reports or case series and therefore associated with an overall high risk of bias. There were only three domains in which reviewed studies scored a low risk of bias. These domains were 'blinding outcome assessment', 'incomplete outcome data' and 'selective reporting'. Studies were only scored a low risk of bias if they were not likely to be influenced by lack of blinding, did not miss any outcome data and did not selectively reported their results.

\section{Procedural data}

In most cases, procedures of ICA dissection treatment and intracranial occlusion treatment were combined to treat both abnormalities effectively. Seven studies described a cervical origin of the ICA dissection, whereas there was one study that described the case of an intracranial ICA dissection. We found that 12 studies exclusively described procedures in which the ICA dissection was addressed first. Furthermore, two studies described a treatment strategy with first addressing the intracranial occlusion before stenting the ICA dissection. In two studies, it was not clearly described which occlusive pathology was addressed first.

\section{Procedures of ICA dissection treatment}

Permanent stent placement in ICA dissection together with antiplatelet therapy was described in ten studies [1, 16-24]. Permanent stents used in these cases were Carotid Wallstent [1, 16, 19, 20, 22, 24], Enterprise stent [17, 21], Wingspan stent [21], Acculink stent [25] and Leo stent [18, 24]. In four studies [2, 26-28], no stents were used to treat the ICA dissections.

\section{Procedures of intracranial occlusion treatment}

Eleven studies showed IA thrombolytics as a supplemental treatment for the intracranial occlusion next to permanent stenting of the ICA dissection [1, 2, 16, 18-21, 23, 25, 27, 28]. In the two studies which did not describe the use of stents to treat the ICA dissection $[27,28]$, one case described treatment of the intracranial occlusion with IA rt-PA [28] and one case described use of IA urokinase as treatment for the intracranial occlusion [27].

One study [2] described the use of a Merci stent retriever to treat the intracranial occlusion. Two studies [25, 26] described the use of a Penumbra thromboaspiration system without a direct aspiration first-pass technique (ADAPT). Treatment of the intracranial occlusion with a Solitaire stent retriever was described in three studies $[22,27,29]$. In most cases, a combination of the approaches which are mentioned above is performed. For a more comprehensive description of performed procedures, see Table 1.

\section{Complications during procedures}

In one case [17], decompressive craniectomy was performed after endovascular therapy and one case described an extension of the dissection caused by a microcatheter [2]. This extension was treated effectively with stenting.

\section{Reperfusion outcome data}

Not all included studies described the reperfusion outcomes. Reperfusion outcome data were described in 16 studies. TICI 2b-3 was described in 10 studies. In only a few studies, TICI 2 a was reported after recanalisation. Even less studies presented patients with very limited reperfusion. For individual TICI data, see Table 1.

\section{Clinical outcome data}

As displayed in Table 1, most studies showed favourable clinical outcome and successful reperfusion. Eleven out of 12 studies that described clinical outcome using 90-day mRS showed favourable clinical outcome. Seven out of eight studies that described 90-day NIHSS as an outcome parameter showed a favourable clinical outcome.

\section{Discussion}

Most included studies on endovascular treatment in patients with proximal intracranial occlusion and a concomitant ICA dissection present favourable clinical outcome and successful reperfusion. A favourable 90-day NIHSS was found in the majority of cases in which this parameter was denoted. Besides, favourable 90-day mRS was found for most of the cases for patients of which the follow-up was classified by mRS. The vast majority of treated patients had successful reperfusion. However, it should be noted that the risk of bias in these studies is high.

A similar study [30] indicated that endovascular treatment had more beneficial outcomes than intravenous treatment in patients with ICA dissection-related stroke. Yet, in this study, there was no differentiation between patients with an exclusive ICA dissection and patients with both an ICA dissection and concomitant occluded intracranial artery. The fact that this study also included patients with an ICA dissection without an additional intracranial occlusion might explain why they found a higher percentage of patients with favourable reperfusion. The percentage of cases with favourable clinical outcome was comparable to our series.

Another recent study on RECOST study data [24] showed that a distal to proximal treatment strategy of ICA dissection with a concomitant intracranial occlusion appears safe and effective [24]. It is argued in this study that a conservative 


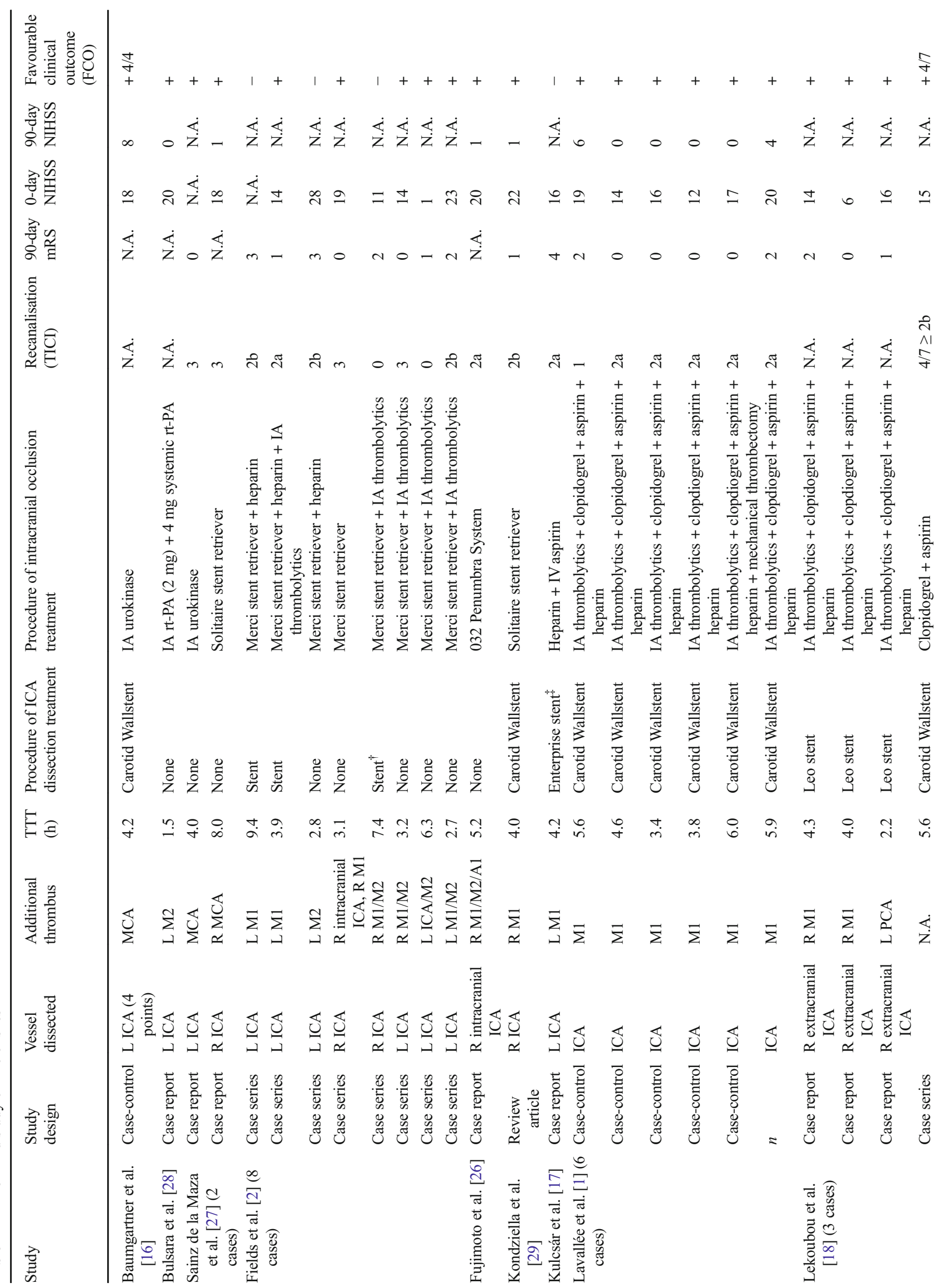




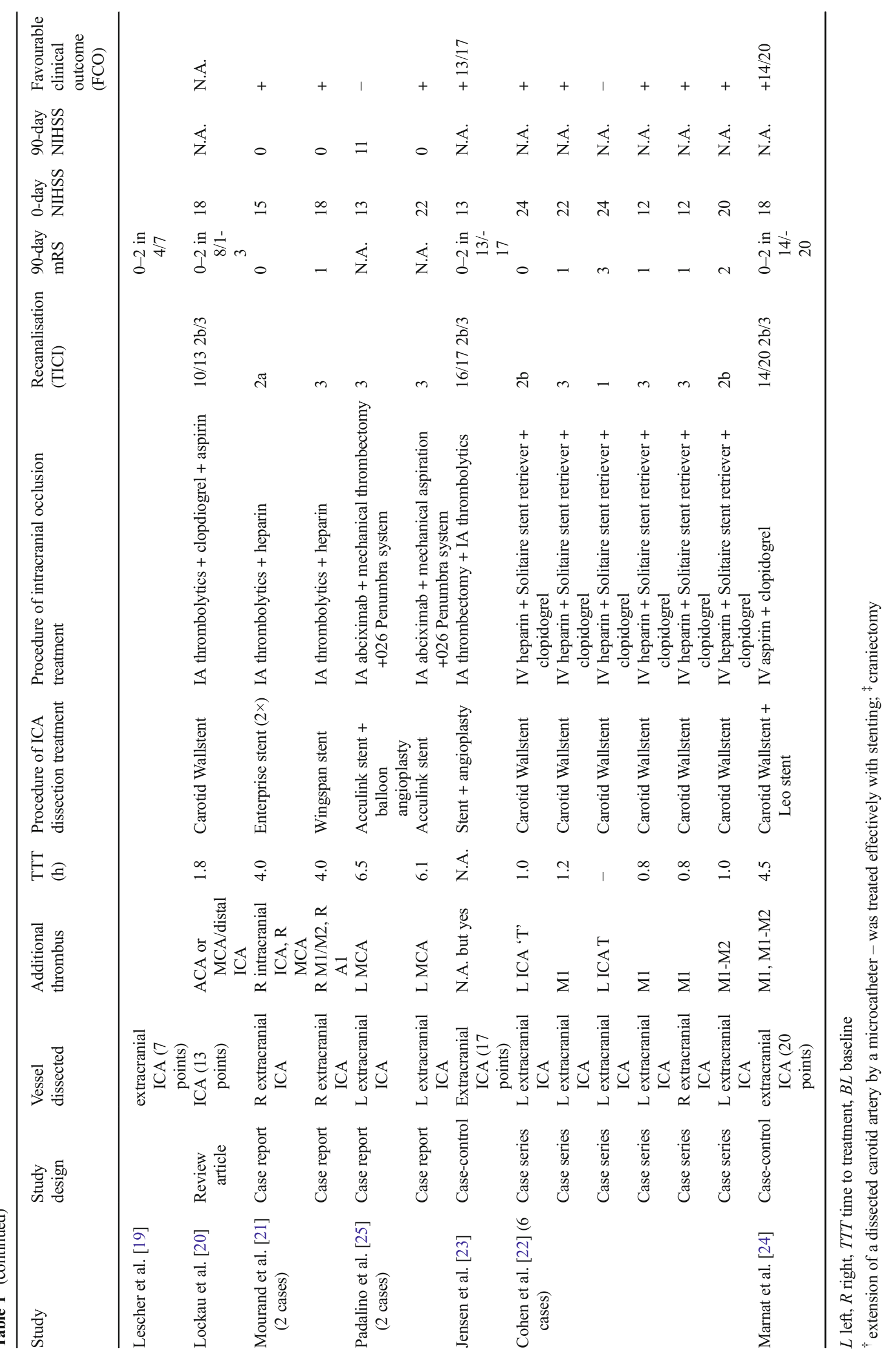


approach (considering stent placement only after intracranial revascularisation in case of circle of Willis insufficiency) may be reliable and safe. However, the RECOST study included only 20 patients with an ICA dissection together with an intracranial occlusion. Hence, beneficial outcome of a conservative approach has only been researched in a small number of patients.

Regarding the risk of bias of included studies, most of the included studies were retrospective case reports or case series (see Table 1). By definition, this led to a high sensitivity to bias. It is possible that only cases with favourable clinical outcome rates and successful reperfusion were published. This could also explain why outcomes found in our review are substantially more positive than the outcomes in the held RCTs [6-12].

Data in the included studies was not always consistently described. Some studies only used mean values of all included patients and patient characteristics were not always clearly described. This could have influenced the accuracy of our results, since individual results are left out.

Only a small number of patients received treatment of the intracranial occlusion with second-generation thrombectomy devices (Solitaire or Penumbra thromboaspiration system). All other included cases described the use of currently obsolete techniques. Unfortunately, studies that describe results of secondgeneration treatment methods are not widely available yet.

Furthermore, time to treatment in the reviewed studies was relatively long. This could be due to the fact that the procedural time that is needed to stent the ICA increases the onset-toreperfusion time. This applies for a treatment strategy with first performing recanalisation in the ICA and secondly in the intracranial occlusion. The relatively long TTT could possibly be explained by the fact that most patients were initially not presented to the hospital with a dissection. The additional finding of ICA dissection in the emergent setting and the subsequent need for an experienced endovascular therapist might have delayed the procedure. No statistics on the collected data could be performed since the patient characteristics and data were non-poolable. This is because included patients did not have similar baseline characteristics, different types of treatment were used, and data measurement varied between studies.

The high success rates reported in the included studies suggest that endovascular treatment is safe and efficacious for patients with a proximal occlusion with a concomitant ICA dissection. However, we need to stress that the number of patients included in this review is low and the risk of bias is high. Therefore, further research is needed.

\section{Conclusion}

High rates of favourable outcome and reperfusion have been presented in reviewed studies. This suggests that endovascular treatment is beneficial in patients with ICA dissection and concomitant proximal intracranial occlusion. This corresponds with earlier emerged studies that showed that mechanical thrombectomy is beneficial in patients with large vessel occlusion. However, this review has only taken data of a limited group of patients into account. A pooled analysis of patients with ICA dissection and concomitant proximal intracranial occlusion from the recently published endovascular treatment trials and running registries is therefore recommended.

\section{Compliance with ethical standards}

Funding No funding was received for this study.

Conflict of interest The authors declare that they have no conflict of interest.

Ethical approval For this type of study formal consent is not required.

Informed consent Statement of informed consent was not applicable since the manuscript does not contain any patient data.

Open Access This article is distributed under the terms of the Creative Commons Attribution 4.0 International License (http:// creativecommons.org/licenses/by/4.0/), which permits unrestricted use, distribution, and reproduction in any medium, provided you give appropriate credit to the original author(s) and the source, provide a link to the Creative Commons license, and indicate if changes were made.

\section{References}

1. Lavallee PC, Mazighi M, Saint-Maurice JP et al (2007) Stentassisted endovascular thrombolysis versus intravenous thrombolysis in internal carotid artery dissection with tandem internal carotid and middle cerebral artery occlusion. Stroke 38(8):2270-2274. doi: 10.1161/strokeaha.106.481093

2. Fields JD, Lutsep HL, Rymer MR et al (2012) Endovascular mechanical thrombectomy for the treatment of acute ischemic stroke due to arterial dissection. Interv Neuroradiol 18(1):74-79

3. Kim DJ, Kim DI, Byun JS et al (2008) Intra-arterial thrombolytic therapy for hyperacute ischemic stroke caused by tandem occlusion. Cerebrovasc Dis 26:184-189. doi:10.1159/000145326

4. Machi P, Lobotesis K, Maldonado IL et al (2012) Endovascular treatment of tandem occlusions of the anterior cerebral circulation with solitaire FR thrombectomy system. Initial experience. Eur J Radiol 81(11):3479-3484. doi:10.1016/j.ejrad.2011.12.032

5. Liebeskind, DS et al. Spontaneous cerebral and cervical artery dissection: treatment and prognosis. In: UpToDate, Post TW (Ed), UpToDate, Waltham, MA. http://www.uptodate.com/contents/ spontaneous-cerebral-and-cervical-artery-dissection-clinicalfeatures-and-diagnosis (Accessed on November 30, 2016.)

6. Berkhemer OA et al (2015) A randomized trial of intraarterial treatment for acute ischemic stroke. N Engl J Med 372:11-20. doi:10. 1056/NEJMoa1411587

7. Goyal M, Demchuk AM, Menon BK et al (2015) ESCAPE trial investigators. Randomized assessment of rapid endovascular treatment of ischemic stroke. N Engl J Med 372:1019-1030

8. Jovin T, Chamorro A, Cobo E et al (2015) REVASCAT trial investigators. Thrombectomy within 8 hours after symptom onset in ischemic stroke. N Engl J Med 372:2296-2306 
9. Saver JL, Goyal M, Bonafe A et al (2015) SWIFT PRIME investigators. Stent-retriever thrombectomy after intravenous t-PA vs. tPA alone in stroke. N Engl J Med 372:2285-2295

10. Campbell BCV, Mitchell PJ, Kleinig TJ et al (2015) EXTEND-IA investigators. Endovascular therapy for ischemic stroke with perfusion-imaging selection. N Engl J Med 372:1009-1018

11. Bracard $\mathrm{S}$ et al (2016) Mechanical thrombectomy after intravenous alteplase versus alteplase alone after stroke (THRACE): a randomised controlled trial. The Lancet Neurology 15(11):11381147

12. Muir KW et al (2016) Endovascular therapy for acute ischaemic stroke: the Pragmatic Ischaemic Stroke Thrombectomy Evaluation (PISTE) randomised, controlled trial. J Neurol Neurosurg Psychiatry. doi:10.1136/jnnp-2016-314117

13. Fugate JE, Klunder AM, Kallmes DF (2013) What is meant by "TICI"? AJNR Am J Neuroradiol 34(9):1792-1797. doi:10.3174/ ajnr.A3496

14. Higgins, JPT, Altman, DG (editors) (2015); Chapter 8: assessing risk of bias in included studies. In: Higgins JPT, Green S (editors). Cochrane Handbook for Systematic Reviews of Interventions Version 5.0.1 (updated September 2008). The Cochrane Collaboration, 2008. Available from www.cochrane-handbook.org

15. Young FB et al (2005) Comparison of the National Institutes of Health stroke scale with disability outcome measures in acute stroke trials. Stroke 36:2187-2192

16. Baumgartner RW, Georgiadis D, Nedeltchev K et al (2008) Stentassisted endovascular thrombolysis versus intravenous thrombolysis in internal carotid artery dissection with tandem internal carotid and middle cerebral artery occlusion. Stroke 39(2):e27-e28. doi:10. 1161/strokeaha.107.500959

17. Kulcsar Z, Bonvin C, Lovblad KO et al (2010) Use of the enterprise (TM) intracranial stent for revascularization of large vessel occlusions in acute stroke. Clin Neuroradiol 20(1):54-60. doi:10.1007/ s00062-010-9024-x

18. Lekoubou A, Cho TH, Nighoghossian N et al (2010) Combined intravenous recombinant-tissular plasminogen activator and endovascular treatment of spontaneous occlusive internal carotid dissection with tandem intracranial artery occlusion. Eur Neurol 63(4):211-214. doi: $10.1159 / 000278248$

19. Lescher S, Czeppan K, Porto L et al (2015) Acute stroke and obstruction of the extracranial carotid artery combined with intracranial tandem occlusion: results of interventional revascularization. Cardiovasc Intervent Radiol 38(2):304-313. doi:10.1007/s00270014-1047-2
20. Lockau H, Liebig T, Henning $\mathrm{T}$ et al (2015) Mechanical thrombectomy in tandem occlusion: procedural considerations and clinical results. Neuroradiology 57(6):589-598. doi:10.1007/ s00234-014-1465-5

21. Mourand I, Brunel H, Vendrell JF et al (2009) Endovascular stentassisted thrombolysis in acute occlusive carotid artery dissection. Neuroradiology 52:135-140

22. Cohen JE, Gomori JM, Rajz G et al (2014) Extracranial carotid artery stenting followed by intracranial stent-based thrombectomy for acute tandem occlusive disease. J Neurointerv Surg. doi:10. 1136/neurintsurg-2014-011175

23. Jensen J et al (2016) Comprehensive analysis of intra-arterial treatment for acute ischemic stroke due to cervical artery dissection. J NeuroIntervent Surg. doi:10.1136/neurintsurg-2016-012421

24. Marnat $\mathrm{G}$ et al (2016) Endovascular management of tandem occlusion stroke related to internal carotid artery dissection using a distal to proximal approach: insight from the RECOST study. AJNR Am J Neuroradiol 37:1281-1288

25. Padalino DJ, Deshaies EM (2012) Tandem middle cerebral arteryinternal carotid artery occlusions: reduced occlusion-torevascularization time using a trans-anterior communicating artery approach with a penumbra device. J Neurosurg 116(3):665-671. doi:10.3171/2011.10.jns111516

26. Fujimoto M, Tateshima S, Ali L et al (2013) Direct thrombus aspiration using the penumbra system for the treatment of pediatric intracranial dissection. J Neurointerv Surg 5(6):e43. doi:10.1136/ neurintsurg-2012-010474.rep

27. Sainz de la Maza S, De Felipe A, Matute MC et al (2014) Acute ischemic stroke in a 12-year-old successfully treated with mechanical thrombectomy. J Child Neurol 29(2):269-273. doi:10.1177/ 0883073813509889

28. Bulsara KR, Ediriwickrema A, Pepper J et al (2013) Tissue plasminogen activator via cross-collateralization for tandem internal carotid and middle cerebral artery occlusion. World J Clin Cases 1(9):290-294. doi:10.12998/wjcc.v1.i9.290

29. Kondziella D, Cortsen M, Eskesen V et al (2013) Update on acute endovascular and surgical stroke treatment. Acta Neurol Scand 127(1):1-9. doi:10.1111/j.1600-0404.2012.01702.x

30. Haussen DC, Jadhav A, Jovin T et al (2015) Endovascular management vs intravenous thrombolysis for acute stroke secondary to carotid artery dissection: local experience and systematic review. Neurosurgery. doi:10.1227/neu.0000000000001072 